\title{
ENME: An ENriched MEdia Application Utilizing Context for Session Mobility; Technical and Human Issues
}

\author{
Egil C. Østhus ${ }^{1}$, Per-Oddvar Osland ${ }^{2}$, and Lill Kristiansen ${ }^{1}$ \\ ${ }^{1}$ Dept. of Telematics, NTNU, Norw. Univ. of Science and Technology, \\ O.S. Bragstads Plass 2A, NO-7491Trondheim, Norway \\ \{egilconr, lillk\}@item.ntnu.no \\ ${ }^{2}$ Telenor R\&D, Otto Nielsens vei 12, N-7004 Trondheim \\ per-oddvar.oslandatelenor.com
}

\begin{abstract}
We look into the combination of a SIP application (IP based multimedia telephony) together with a context-aware smart environment. We start by describing a scenario where it is highly relevant to use such a combination. The combined application is called ENME, and is managing and moving communication sessions based on user context. We are realizing the service with SIP REFER and SIP extensions. We also discuss briefly other solutions such as 'virtual terminals', and we identify pros and cons of the different solutions. The application is implemented, and runs on a model railroad system, but the context model itself is more general. A next step would be to deploy the application in other smart environments, and we look briefly into a hospital environment. We end the paper by identifying some human issues for the service to work properly, and relate these issues to the technical solutions.
\end{abstract}

\section{Introduction}

Pervasive computing, a term long spoken of, now seems to slowly become a reality. In visionary descriptions, pervasive computing has been depicted as an environment where computing resources are integrated into more or less every device and physical object. These resources are naturally online, mobile and communicating to fulfill specific tasks.

In our paper we combine pervasiveness and context with an IP multimedia telephony application realized by the SIP protocol from IETF. We focus on a (value added) service ENME that manage communication sessions based on user context.

A scenario illustrates the use of such an application: A (human) user moves from a low capability zone into a high capability zone during an ongoing communication session. (The movement may be detected with various location technologies.) The new zone has higher capacity such as a (bigger) screen, video possibilities and so forth. We assume that the user is alerted about the new features and decides to move (parts of) his ongoing session to this new device(s).

The scenario serves as a basis for an implementation of an application realizing one of the solution proposals. The application is given the name ENME. Our implementation is done on a model railroad system in order to simulate mobility between zones, but here we illustrate the use of this application in a hospital setting. 


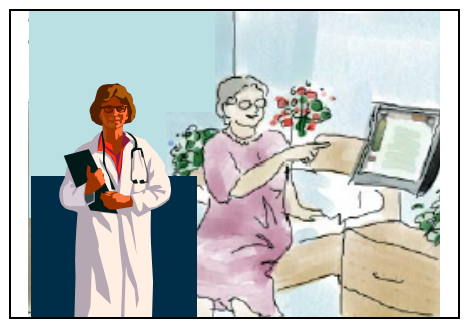

Fig. 1. The physician utilizes a bigger screen when entering a patient room

We will look into relevant background work from pervasive computing and context awareness, and combine this with the IP-based multimedia telephony. We will look into different ways to implement the application. The different solutions will be briefly compared. In this paper we identify some issues relating to human factors. We lookinto those issues having implications on the technical realization in terms of SIP.

We will end the paper by illustrating how ENME may be used in a hospital scenario and some human factors of particular importance.

\section{Former Work on Pervasiveness and Context}

Based on the previous scenario, the following are identified as relevant background material and former work on pervasiveness and context. In the later sections this material will be linked to our implementation and discussion.

\subsection{Pervasive Computing}

Much work on mobile computing, ubiquitous and pervasive computing comes from computer science research.. We have our background in (mobile) telematics / telecommunication, and to us live audio, video and continuous handover of such sessions are natural to look into. In a converged manner we also look into endpoint capable of both 'computing' and 'communication'.

We may note that ICT can be invisible in several ways, e.g. by being integrated into other devices such as refrigerators, eye wear (glasses) etc, or by being mentally integrated as a natural human tool.

Satyanarayanan [1] points out four research thrusts in connection with pervasive computing:

Effective use of smart spaces. A space is a meeting room, a corridor or a welldefined area.

Invisibility. Weiser's ideal that the computers disappear from the users' consciousness.

Masking Uneven Conditioning. The deployment of pervasive computing into the environment is depended on non-technical factors such as organizational structure, business models and economics. 
Localized Scalability. The number of smart spaces increases, The computational power in those spaces increases as well. The presence of multiple users will further complicate the problem. [1] regards scalability as a problem in pervasive computing. Coming from more of a telecom side we might reformulate it as an issue to be solved. (Telecom has a long history in handling scalability.)

For a more detailed overview of pervasive computing and related topics, see e.g. Satyanarayanan [1].

\subsection{Context}

In a pervasive environment, context awareness is an important factor. At the same time, the definition of context is somewhat unclear. There have been several papers published, either trying to define context awareness or presenting new models for context. When this is said, no standards have been proposed. In this paper we use a definition proposed by Dey [2].

"Context is any information that can be used to characterize the situation of an entity. An entity is a person, or object that is considered relevant to the interaction between a user and an application, including the user and application themselves."

This definition points out that both the application and the user is important when it comes to context-aware computing. In addition, it points out that the device executing the application is important as well as the nearby devices.

\subsubsection{Managing Context Info}

Using the above definition, it is obvious that context spans over a wide area of information. Mostéfaoui et al [3] suggest splitting the context into three categories: Sensed context, Derived context and Explicitly provided context.

\begin{tabular}{|c|}
\hline Fusion \\
\hline Conversion \\
\hline Measurements \\
\hline Sensor \\
\hline
\end{tabular}

Fig. 2. The context stack [4]

To ease the handling for the context information, $\mathrm{Li}$ [4] propose a context stack as shown in Figure 2. The different characteristics of the context information are taken into account in this model. This layering model makes it possible to handle the fact that context information has many alternative representations. The context application using this context stack does not need to know about this, a well-defined interface between the stack and the application hides such details. The context stack is also suitable for derived context, if the sensor layer is thought of as a logical sensor.

\subsubsection{Context Models}

When the context is to be incorporated as a part of a computing environment, the context entities have to be represented in a manner suitable to the execution logic. Different models have been proposed. E.g. Henricksen et al [5] introduce an object- 
oriented approach. They suggest dividing the context information into persons, devices and channels. The association between these entities is further subdivided into static and dynamic associations. (See also Figure 3(right part) in section 4.2 for details of their model)

\section{Enabling Technologies}

This section gives a brief overview of some technologies relevant for realizing context-based session management.

One may envision that a high capacity zone may be determined either by some sort of location technology, or some sort of service discovery protocol. We refer to the overview paper by Helal [9] for more information on service discovery.

The positioning used in our implementation is based on Radio Frequency Identification (RFID).

The rest of this section a brief introduction to SIP and SDP. More info on SIP may be found e.g. in the book [6].

Session Initiating Protocol (SIP) is a protocol used to establish and maintain a session. SIP is defined by IETF in RFC 3261[7], often referred to as "Baseline SIP". The basics components in SIP are the SIP UserAgent (UA), SIP Proxy, SIP Registrar server and SIP redirect server. The user is typically interacting with the SIP UA. The user is identified with a SIP address, that looks just like an email address “sip:userA@item.ntnu.no". When a user wants to use a SIP UA, he has to register himself with the SIP Registrar. Dialog is a key concept in SIP. One or more dialogs can be a part of a single session.

Session Description Protocol (SDP) [8] provides the receiver with information about the multimedia session and makes the receiver able to participate in the session if desired. A multimedia session consists of a set with media streams that has certain duration. SDP is carried within an SIP message, and typically describes session name and purpose, time(s) the session is active, the media comprising the session, and information to receive those media (addresses, ports, formats and so on).

\section{The Design of the ENME (Enriched Media) Application}

The design of the ENME application is based on the work and principles as presented in sections 2 and 3.

In this section we start by presenting a minimal yet adequate model for applications that manage communication sessions based on user context. Then we present the ENME service and the realized system.

\subsection{Entities and Relations}

Our model consists of a set of basic entities: User, Zone, Device and Session. These entities are described in Table 1 and the relations between them are sketched in Figure 3.

Communication is between users, the technical part of the communication being handled by devices. Users move between various zones, and the access to devices may change when a user moves to a new zone. Devices may be stationary within a 
zone, or may move from zone to zone. A user or device may be in one and only one zone (at a time). A zone may have zero or more users and devices. In our implemented system we assume that all users in a zone have access to all devices in that zone, but authorization mechanisms can be supplied to relax this assumption.

Table 1. Entities

\section{Entity Comment}

\begin{tabular}{|l|l|}
\hline User & A person with access to use the offered application \\
\hline Zone & $\begin{array}{l}\text { A geographical area, e.g. a room or inside a booth. A user and a device } \\
\text { are located inside a zone. }\end{array}$ \\
\hline Device & $\begin{array}{l}\text { A terminal, both public and nonpublic available. A device is described } \\
\text { by its capabilities (ability to support video and voice, screen resolution, } \\
\text { speakers, related codecs, etc). Sub-entities: High/low capability device. } \\
\text { (In the implementation only available codecs are looked at.) }\end{array}$ \\
\hline Session & $\begin{array}{l}\text { A session is a communication between two or more parties. A session } \\
\text { may consist of zero or more dialogs. A dialog will comprise media } \\
\text { transfer. A zero-dialog session consists of only signaling, but no media } \\
\text { transfer. This is according to SIP [7], and is further described in 0 }\end{array}$ \\
\hline
\end{tabular}

The data model in Figure 3 (left) excludes the details about available attributes. For sessions, it is important to have information about both the requested (wanted) media description, and those currently used. These values are available in the SDP message (but the wanted description may not be kept by the endpoint (UA), hence we need to keep it in our context model). In addition is it important to include the session identifier. Its capabilities and the zone it currently is within describe the devices. Contact information is also important information. The session information is dynamically updated as sessions are accepted and terminated.

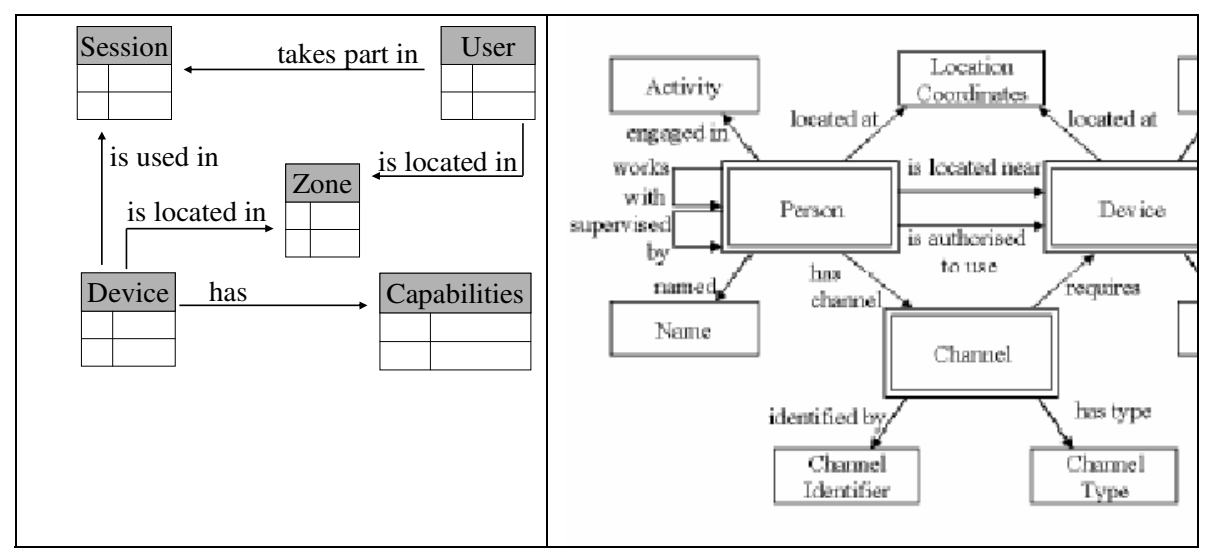

Fig. 3. Entities and relations. Left: Our model. Right: The model from [5]. We may roughly compare user - person and session - channel. 


\subsection{ENME: Service (Application) Logic}

The main objective behind the model is to create an application that manages communication sessions based on context. More precisely, we want to take into account the devices a user has available, and engage the session at the device that best suits the users' requirements.

As the user move, device availability may change. (We assume availability of 'public' high capability devices available in the user's environment.) The model should also facilitate session mobility, i.e. to move the session to a new device if it is better suited than the one currently in use. To decide if a newly available device is better suited than then ones in use, it is necessary to keep overview of service descriptions requested at the beginning of the session. This leads to a service logic as briefly described in Table 2 .

Table 2. Service logic
0. Assume there is an ongoing communication session between two or more users, the service maintain knowledge about users preferences when it comes to service description, e.g. voice only or video. devices that are better than the ones in use, and match user's preferences, proceed to Step 2. Otherwise return to Step 0.
1. If a user moves to a new location, check for available devices. If there are
2. Request the user if he/she wants to move the session to a new device. Proceed to Step 3 if positive answer, return to Step 0 otherwise.
3. Move (parts of) the session to new device.

\subsection{System Overview and Design}

A fundamental principle in our implementation has been to use standard protocols for service parts that involve devices, and develop a network-centric service that keeps as much as possible of the developed logic in a controllable environment. This enables us to use off-the-shelf software for terminal-related parts of the service (with some minor modifications). The service itself is located at a centralized application server. A deployment overview is given in Figure 4 (next page).

The context stack described in section 2.2.1 is partly used, as we separate the RFID (sensor) from other context handling. This will enable RFID to be used in many different applications.

REFER [10] is a SIP extension requesting the recipient to refer to a provided source. Call transfer is one service that is enabled by REFER. What makes the use of REFER somewhat complicated for session movement, is the fact that the REFER RFC does not require the originally dialog to be automatically terminated. REFER requests the receiver to contact an additional source, not to actually move an ongoing session. For the session to be moved, it requires the (human) user to active take part by terminating the originally dialog. For session movement it is convenient that the user terminal performs this task automatically. In order to achieve this, it is necessary to be able to specify different ways to use REFER. 


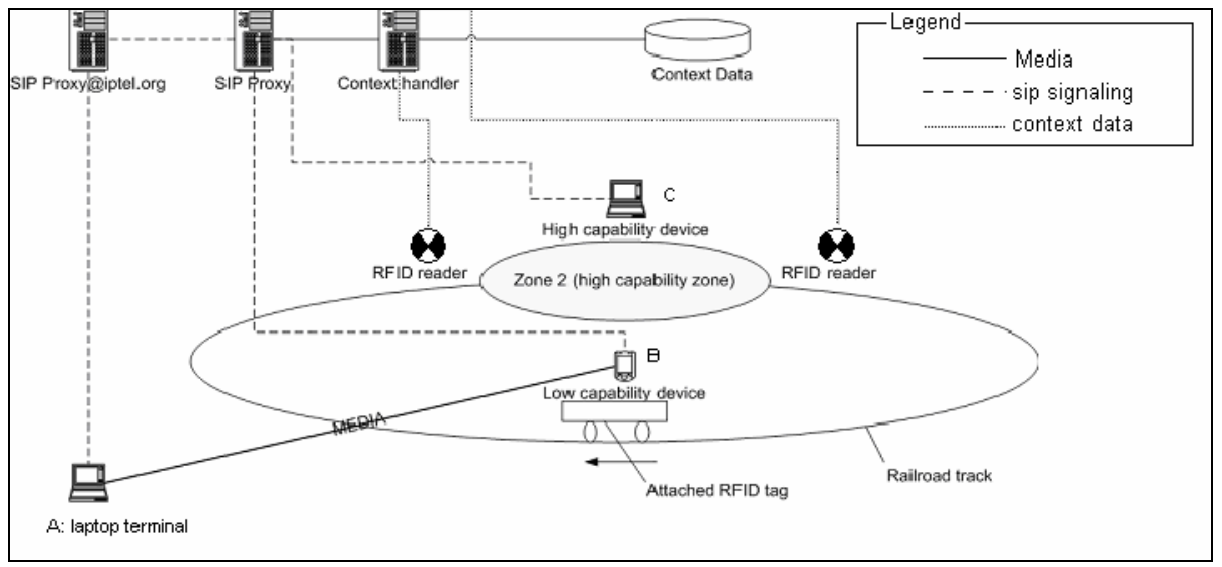

Fig. 4. Deployment overview. The prototype is implemented on a model railroad system (as indicated) $\mathrm{A}, \mathrm{B}$ and $\mathrm{C}$ will be referred to later in the text.

ENME has focus on demonstrating SIP for session management. Issues such as context distribution are left out of this paper. In order to be able to implement the service logic as described in Table 2 various solutions are possible, and one was implemented. (Some alternatives are discussed in section 5.)

In order to involve the (human) subscriber of the ENME service, we found it necessary to introduce a SIP extension (we call it CCRequest). This will be sent to the $\mathrm{B}$ terminal, and will be followed by the SIP REFER (after a dialog window to the real end user. The CCRequest is a SIP extension that requests the receiver to initiate a request.) The SIP extension was needed because current SIP and SIP extensions does not offer this functionality. Details of the SIP flows are left out due to lack of space.

The main steps from Table 2 will be realized as follows: The Context Handler is using a SIP Interface in order to send and receive SIP messages. When the Context Handler is notified that a user has moved to another zone, and if the service logic described in Table 2 executes successfully, it sends a CCRequest to the B terminal in order to notify it of the newly available high capability device. For the ENME application the receiver initiates a REFER message to its corresponding communication partner. After that the message flow is according REFER RFC [10], and the media flow is now between $\mathrm{A}$ and $\mathrm{C}$.

\section{Discussion}

There are several ways to implement the service logic described in step 3 in Table 2 . We will discuss pros and cons for the implemented solution, as well as for some alternatives. Details of the alternatives in terms of SIP messages etc. will not be described here due to lack of space, but we will give some evaluation on some of the solutions. The discussion is focused around human factors. Some technical issues are also mentioned. 


\subsection{The Implemented Solution: Some Discussion}

The implemented solution puts the (human) user communicating using device B in control over the service execution. Since B is the subscriber of the ENME service this makes perfectly sense. The REFER RFC will establish an Event subscription forcing the A terminal to notify B the outcome of the session movement. This way the B user is aware of why the session handover fails if it does. Such information is considered crucial for being able to create a user-friendly service. These are 2 important pros with this solution. On the more technical side: The negative side is that we needed a SIP extension. The positive side is that A only needs to supports the basic SIP/SDP messages and the well established REFER method for ENME to work.

\subsection{Alternative Solution 1: Some Discussion}

This alternative is to have the ContextHandler/SIPinteface sending a REFER message to the A terminal. This raises some problems, and we will describe two of them here. The (human) user communicating using the B terminal is the subscriber of the service, not the user at terminal A (who may in fact be unaware of the existence of ENME application in the general public case). Consequently should the (human) B user be queried whether the session should be moved or not. On the more technical side: Also A (who is not subscribing to this service) will need updated software in this case.

\subsection{Alternative Solution 2 ('Virtual Terminal'): Some Discussion}

'Virtual terminal' is a concept to combine different devices in order to deliver a service that is capable of deliver richer media than any of the devices would manage alone.

One approach is send both video and voice to the low capability device, and have the low capability device forward the video part of the stream to the high capability device using a Personal Area Network (PAN). In this case the low capacity device needs not display the video, but it needs to forward it. This solution puts further requirements to the user terminal, both in term of service discovery and video reception/forwarding.

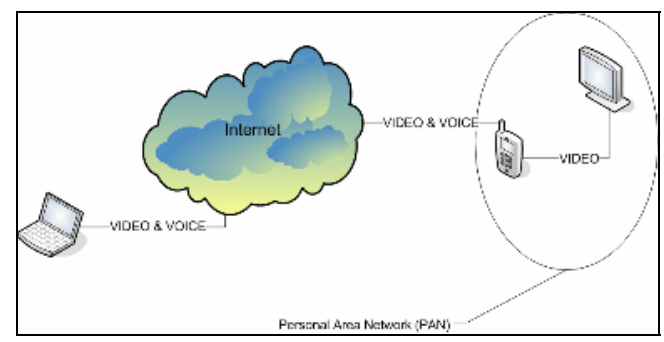

Interactive conversational multimedia showing the colleague's face puts strong requirements both on synchronization and time delay. By creating virtual terminals, problems with synchronization and timing might become an issue. We may notice that this issue does not occur for data applications, but is crucial for many uses of video streams, since it is a fact that lip-out-of-synchronization is more disturbing than no video at all. 
Talking to a colleague and at the same time looking at an independent video stream will however have fewer requirements on the synchronization.

\section{ENME in a Hospital Setting: Some Issues}

We have already indicated (Figure 1) that ENME may be deployed in a hospital setting, via high capacity screens on patient rooms, in corridors, in offices or in special 'multimedia booths'. In these cases video telephony may be relevant, but also cases of voice telephony enhanced with data applications are relevant (like 'joint looking' at a patient journal). It might also be relevant to look at video of say ECG, while having voice conversation to another person. We will now identify some human issues relevant for the ENME service.

But first we will comment on the use of a location infrastructure inside a hospital. Our system uses RFID sensors. As pointed out already in 4.3 our solution allows for many applications to use the same infrastructure. Other applications that may take advantage of location might be drug management, as proposed in [11].

\subsection{User Involvement or User Disturbance?}

The ENME application as described provides help to its users to establish as rich media session as possible at any time. The naïve assumption is that all users always want the richest media available, but this is not necessarily the case. Følstad et al. [12] has studied what criteria were used to decide what communication service to use for different tasks. This field study showed that the users where conscious what communication service was most suitable for each task. Hence it is obvious that adding video to an ongoing communication service may not be wanted in all cases, and our solution supports this. It may also be useful to separate voice-with-possibleenhancement-telephony from voice-and-always-video-telephony. ENME may be used with both, but the disturbance of changing terminal must be considered for the latter, and an ENME on/off button may easily be added.

\subsection{One Terminal or Many Terminals?}

The Knowmobile research project gave PDAs, laptops and GSM-phones to physician students carrying out work in hospitals. In [1] they write: "The multidevice paradigm leads to problems connected to the use, design, harmonization, [...] of various devices". This shows that we are facing challenges in the design of an application like ENME.

Other question like: Shall the GUI for ENME application control move to the bigger screen, or shall it stay on the handheld device at all times? This needs to be studied further, and may require some technical studies (at SIP level) as well.

\section{Conclusions and Further Work}

By adding context awareness to a communication service, it is possible to create an application that informs the (human) user if a more appropriate device is available nearby. The session can in this situation be moved to the best-fitted device upon 
acceptance from the user. SIP and SIP REFER was used to realize this service. We also pointed out that the subscriber of the service has to be involved in the service execution.

We also analyzed some human factors, and found that humans may not always want a pervasive environment, as pervasive may in fact be considered 'invasive'.

In chapter 6 we identify some human issues relating to disturbance and confusion. These issues are important in all cases, but become more important in cases where the communication itself is not the main task, just a tool supporting the real tasks and activities carried out by the users. This is typically the case in a hospital. Thus it seems relevant to put more focus on the tasks that the user is carrying out, not just the (technical) channels and the devices. This fits in with the findings of [12]. Also the relations between the colleagues may be of importance. This leads us to further organizational issues. This is however left out of this paper.

The application is not analyzed when it comes to privacy issues and security. Security is a particular issue in a hospital setting, but we believe it is important also for ENME as a service for personal use in public settings. Thus this needs further work. Organizational and cultural issues must be studied as well, in particular in the hospital setting.

\section{References}

[1] Satyanarayanan, M., "Pervasive Computing: Vision and Challenges", IEEE Personal Communications, August 2001, pp $10-17$

[2] Dey, A. K., "Providing Architectural Support for Building Context-Aware Applications" PhD thesis, College of Computing, Georgia Institute of Technology, 2000

[3] Mostéfaoui, G. K., Pasquier-Rocha, J., Brézillon, P., "Context-Aware Computing: A guide for the Pervasive Computing Community", proceedings of the ICPS'04

[4] Li, Wei, "A Service Oriented SIP infrastructure for Adaptive and Context-Aware Wireless Services", proceedings of the 2nd International Conference on Mobile and Ubiquitous Multimedia, 2003 (Norrköping, Sweden)

[5] Henricksen, K., Indulska, J., Rakotonirainy, A., "Modeling context information in pervasive computing systems", proceedings of Pervasive 2002 (Zürich, Switzerland): 167-180, 2002

[6] Johnston, Alan B. SIP: Understanding the Session Initiation Protocol, Artech House, 2001

[7] Rosenberg, J., Schulzerinne, H., Camarillo, G., Johnston, A., Peterson, J., Sparks, R., Handley, M., Schooler, E., "SIP: Session Initiation Protocol” IETF Networking Group, RFC3261, http://www.ietf.org/rfc/rfc3261.txt

[8] Handley, M., Jacobson V., "SDP: Session Description Protocol", IETF Networking group, RFC 2327, http://www.ietf.org/rfc/rfc2327.txt

[9] Helal, S., "Standards for Service Discovery and Delivery", IEEE Pervasive Computing Vol 1(3), July - September 2002, pp 95 - 100

[10] Sparks, R., "The Session Initiation Protocol (SIP) REFER Method", IETF Networking group RFC 3515, http://www.ietf.org/rfc/rfc3515.txt

[11] Lindeberg, L. and L.Kristiansen, "How a context-aware resource planner for drugs can increase quality in health care" Presented at SHI2005, Aalborg, Denmark, August 2005

[12] Følstad, A. et al., "Fitness-for-Purpose of Person-Person Communication Technologies", IST11577/SEF/DIS/DS/Pub/004/b1, October 31st, 2002

[13] Gallis, H., Kasbo, J. P.and Herstad, J., "The Multidevice Paradigm in Knowmobile Does one size fit all?" Proc. of IRIS24, ed. by Bjørnestad, S. , Moe, R.E., Mørch, A.I. and Opdahl, A.L.. , (2001) pages 491-504 